\title{
An argument for definitional adequacy of RESULT and NARRATION*
}

\author{
Daniel Altshuler \\ Károly Varasdi \\ Heinrich-Heine-Universität Düsseldorf Heinrich-Heine-Universität Düsseldorf
}

\begin{abstract}
Discourse relations link two different discourse units into a compound unit, and it is the presence of such relations that gives a discourse coherence. For example, the relations RESULT and NARRATION are responsible for the perceived narrative progression of the events described in the discourse. Building on Hobbs (1985) and Kehler (2002), we use the definitions of RESULT and NARRATION to derive constraints that pertain to the internal structure of discourse units, and we argue that the plausibility of these constraints lends a new type of support for the definitions that we propose.
\end{abstract}

Keywords: discourse coherence, conditionals, abduction, event structure, definitional adequacy

\section{Introduction}

Discourse coherence theorists assume that there is a primitive class of discourse relations which encode possible ways that ideas could be associated and according to which a discourse is organized, i.e., a discourse is coherent because it is organized by particular discourse relations. Asher \& Lascarides (2003) define discourse relations as edges of a graph whose nodes are discourse units (DUs). DUs come in two types: elementary discourse units (EDUs), which are the atoms of discourse structure, and complex discourse units (CDUs), which are built out of EDUs and may include as few as two or three EDUs, or correspond to multiple pages of text. While Asher and Lascarides (A\&L) do not provide an official definition of EDUs, it is often assumed that each EDU contains a single eventuality description.

This paper is concerned with two discourse relations, RESULT and NARRATION, which A\&L propose are especially relevant for discourses with narrative progression, such as (1) and (2) below.

* We thank the SALT reviewers, organizers and participants for their insight. Thanks also to Sam Cumming, Dag Haug, Zsófia Gyarmathy, Susanna Melkonian and Matthew Stone for providing comments on an earlier version of the draft. This research was supported by the Strategic Research Grant (Strategisches Forschungsfond) from Heinrich-Heine-Universität Düsseldorf for the project Temporal Constraints on Discourse Structure. 
An argument for definitional adequacy of RESULT and NARRATION

(1) a. Max walked up to the light switch.

b. He turned off the light.

a. Max turned off the light.

b. The room became pitch dark.

Observe that in both discourses, the events are understood to have occurred in the order that they appear in the text. Nevertheless, A\&L argue that while the EDUs (1a) and (1b) are connected via NARRATION, the EDUs (2a) and (2b) are connected via RESULT. While A\&L do not offer definitions of these relations, they characterize their causal import (below we represent DUs-i.e., both EDUs and CDUs-with subscripted $\sigma$ 's):

(3) NARRATION $\left(\sigma_{1}, \sigma_{2}\right)$ : The event described in $\sigma_{2}$ is a consequence of (but not strictly speaking caused by) the event described in $\sigma_{1}$ (Lascarides \& Asher 1993: 2).

(4) $\operatorname{RESULT}\left(\sigma_{1}, \sigma_{2}\right)$ : The event described in $\sigma_{1}$ caused the event or state described in $\sigma_{2}$ (Lascarides \& Asher 1993: 2).

Applying these characterizations to the discourses above, A\&L would claim that (1a) and (1b) are connected via NARRATION since the turning off the light is a consequence of (but not strictly speaking caused by) Max walking up to the light switch. On the other hand, (2a) and (2b) are connected via RESULT since the turning off of the lights is understood to have caused the room to become pitch dark.

In sum, A\&L propose that narrative progression often involves two different flavors of causality, encoded by RESULT and NARRATION. This view has been challenged by Kehler (2002), who builds on Hobbs's (1990) proposal that discourse relations are rooted in David Hume's principles of idea association. ${ }^{1}$ In particular, Kehler proposed that the Humean notion of temporal contiguity is characterized by the discourse relation OCCASION, while the Humean notion of cause/effect is characterized by RESULT:

Definition 1 (OCCASION by Hobbs)

Given DUs $\sigma_{1}, \sigma_{2}$, OCCASION $\left(\sigma_{1}, \sigma_{2}\right)$ holds iff

i. A change of state can be inferred from the assertion of $\sigma_{1}$, whose final state can be inferred from $\sigma_{2}$.

ii. A change of state can be inferred from the assertion of $\sigma_{2}$, whose initial state can be inferred from $\sigma_{1}$ (Kehler (2002: 22), citing Hobbs (1985: 10)).

1 Hume famously wrote: "To me there appear to be only three principles of connection among ideas, namely Resemblance, Contiguity in time or place, and Cause or Effect” (Hume 2008: 16). 
Definition 2 (RESULT by Kehler)

Given DUs $\sigma_{1}, \sigma_{2}$, RESULT $\left(\sigma_{1}, \sigma_{2}\right)$ holds iff $P$ can be inferred from the assertion of $\sigma_{1}$ and $Q$ from $\sigma_{2}$, where normally $P \rightarrow Q$ (Kehler 2002: 20).

Notice that Definition 1 and Definition 2 differ in several respects from (3) and (4), respectively. Not only is the notion of causation absent from Definition 1 and Definition 2, but Definition 2 is defined directly in terms of a conditional connecting two propositions, while Definition 1 is characterized in terms of a fine-grained event structure, appealing to changes and their intial states and final states. ${ }^{2}$

In the next section, we will consider Definitions 1 and 2 in more detail. We will argue that NARRATION can, and should, be extracted from Hobbs's (1985) OCCASION, while RESULT can, and should, be built on a refined version of Kehler's (2002) Definition 2 above. In particular, using Definition 1 and Definition 2 as starting points, we define NARRATION and RESULT in terms of enthymematic entailment, inspired by the Support Theories of conditionals (Bennett 2003). With these results in place, we offer in $\S 3$ a novel, indirect method for testing the adequacy of our definitions: Given our definitions and a few natural assumptions, we derive general constraints pertaining to possible EDUs - defined in terms of the event components described in Definition 1-by an abductive argument. We verify the plausibility of our derived EDUs by showing that they correspond to well attested English sentences.

\section{NARRATION and RESULT}

\subsection{Hobbs (1985) on OCCASION}

Hobbs (1985) considers several discourses that he claims exemplify OCCASION. One such discourse involves giving directions:
a. Walk out the door of this building.
b. Turn left.
c. Go to the corner.

In Table 1 we replicated the table in Hobbs (1985) that describes the relevant states and state transitions that occur in discourse (5). The column named "Type" specifies which of the two clauses in Definition 1 is applicable; $l_{1}, l_{2}$ and $a_{1}, a_{2}$ symbolize two kinds of states: the location and the angle of the spatial orientation that the addressee assumes after performing the directions (5a), (5b), and (5c). Finally, the ordered pairs describe a change of state entailed by the performance of the event

2 In subsequent work, A\&L characterize the spatio-temporal import of NARRATION in a way that is similar to Definition 1; see Asher \& Lascarides (2003: 163). 
An argument for definitional adequacy of RESULT and NARRATION

\begin{tabular}{cccc} 
Type & $(5 \mathrm{a})$ & $(5 \mathrm{~b})$ & $(5 \mathrm{c})$ \\
\hline & & & \\
1 & $\left\langle l_{1}, l_{2}\right\rangle$ & $l_{2}$ & \\
2 & & $l_{2}$ & $\left\langle l_{2}, l_{3}\right\rangle$ \\
2 & $a_{1}$ & $\left\langle a_{1}, a_{2}\right\rangle$ & \\
1 & & $\left\langle a_{1}, a_{2}\right\rangle$ & $a_{2}$ \\
\hline
\end{tabular}

Table 1 The relevant states and changes of state in discourse (5)

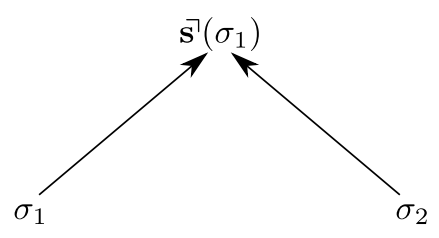

(a) Type 1 pattern

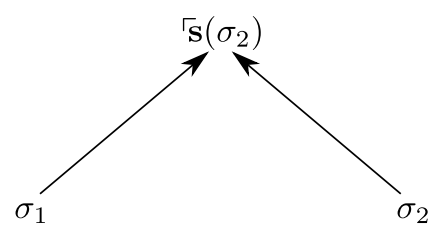

(b) Type 2 pattern

Figure 1 The two disjunctive patterns in Hobbs (1985)

described in the respective EDUs (5a), (5b), and (5c).

For example, performing the event in (5a), the spatial location of the addressee changes from $l_{1}$ to $l_{2}$, while the angle of her orientation is $a_{1}$. Performing $(5 \mathrm{~b})$ doesn't change the state of location of the addressee, but it changes her angle of orientation, and so on.

Note that while performing (5a) entails the change of state $\left\langle l_{1}, l_{2}\right\rangle$, what is crucial with respect to OCCASION is the inference that the addressee is now in location $l_{2}$, which is a sine qua non condition for performing (5c). Put it differently, the fact that (5a) has been completed entails state $l_{2}$ which is the same state that is entailed by the fact that $(5 \mathrm{c})$ has been initiated. Let us call this state $l_{2}$ a pivotal state. The difference between the Type 1 and the Type 2 conditions in the characterization of OCCASION concerns the role that the pivotal state plays: we find the Type 1 pattern when the pivotal state is a final state, and we find the Type 2 pattern when it is an initial state.

Let us introduce the symbols $\overline{\mathbf{s}}$ and $\mathbf{s}$ to denote initial states and final states, respectively. With these symbols we can represent the two patterns in Hobbs (1985) as shown in Figure 1. Note that the arrows represent entailment ("inference") between (the assertion of the) units and (the occurrence of) the respective states. 


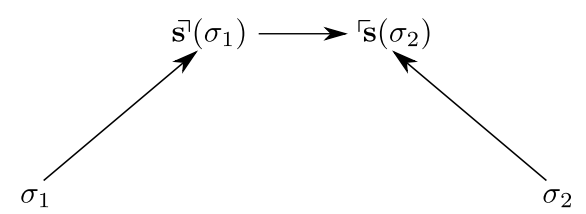

Figure 2 The two patterns generalized

A minimal generalization of the disjunctive patterns We propose a minimal generalization of the two patterns used by Hobbs (1985) that subsumes both patterns under appropriate conditions. The pattern that we propose is in Figure 2.

As can be seen, we suggest inserting a link of implication between the final state $\overrightarrow{\mathbf{S}}\left(\sigma_{1}\right)$ of the first unit and the initial state $\overline{\mathbf{s}}\left(\sigma_{2}\right)$ of the second unit. The two patterns in Hobbs (1985) are then subcases of this more general pattern obtainable by the identification of $\overline{\mathbf{s}}\left(\sigma_{2}\right)$ with $\mathbf{\mathbf { s }}\left(\sigma_{1}\right)$ (Type 1 pattern) or by the identification of $\mathbf{s}\left(\sigma_{1}\right)$ with $\left\ulcorner\mathbf{s}\left(\sigma_{2}\right)\right.$ (Type 2 pattern).

Of course, Figure 2 is not the only possibility to generalize the patterns in Hobbs (1985). Indeed, we could choose the inverse direction of entailment and still have a perfectly viable generalization. However, in Altshuler \& Varasdi In review we show that the inverse direction of entailment is not a viable option because it has consequences that are inconsistent with English data and therefore cannot be endorsed.

\subsection{The definition of NARRATION}

Based on the discussion in the previous section, we offer the following informal definition of NARRATION:

Definition 3 (NARRATION-informal) NARRATION holds between two DUs $\sigma_{1}$ and $\sigma_{2}$ if the occurrence of the final state of $\sigma_{1}$ entails the occurrence of the initial state of $\sigma_{2}$ (see Figure 2).

Before making Definition 3 more explicit, we need to make some further assumptions. Notice the definite description in Definition 3 (and, indeed, in Definition 1). It denotes the final state and the initial state, respectively. This raises the question of how to identify the final state and the initial state that Definition 3 (and Definition 1) refers to. After all, any event, say, going in front of the building (as in Hobbs's (1985) example) has infinitely many ramifications and infinitely many initial conditions that are required to hold in order for the event to be able to start. Which subsets of these infinite sets should we take into account? 
An argument for definitional adequacy of RESULT and NARRATION

We assume that the linguistically relevant final states of discourse units are what Moens \& Steedman (1988) call consequent states. According to Moens \& Steedman, a consequent state of an event "does not necessarily include all events that are objectively and in fact consequences. It rather includes only those consequences that the speaker views as contingently related to other events that are under discussion." For example, we take that (6b) describes a consequent state of (6a):

(6) a. John went into the shop.

b. John was in the shop.

(6b) is indeed a consequence of (6a), i.e., whenever (6a) is true, then (6b) is also true, but not any causal or logical consequence of an event counts as a consequent state. In particular, Moens \& Steedman argue that the English perfect relies on the successful identification of a consequent state without which it is not felicitous; their example is

\# The star has twinkled

where "the hearer cannot identify any relevant consequences," and the perfect is infelicitous. Note that this argument also provides a kind of linguistic diagnostic for final states.

As for initial states, however, the situation is different. For example, in discourse (8), intuitively exemplifying NARRATION,

a. John went into the shop.

b. He bought a Coke.

we assume, in accordance with what we have just said, that the linguistically relevant final state entailed by (8a) is that John is in the shop. This condition entails-in the sense of entailment that we will clarify below-another set of conditions such as that John is within reach of bottles of Coke, he is close enough to the personnel selling the things in the shop, etc. Since these are all conditions that are required for the event in (8b) to get started, the conditions of NARRATION are satisfied.

However, as it stands, Definition 3 is threatened by being vacuously satisfiable in almost every case. For example, John's buying a Coke requires that John exists, and John's being in the shop entails that John exists, so the linguistically relevant final state of (8a), i.e., that John is in the shop, trivially entails a precondition of (8b). This pattern is so general that it makes Definition 3 useless.

Non-trivial preconditions In order to overcome this problem, we refine Definition 3 above as follows. We propose that NARRATION holds between two DUs if the occurrence of the final state of the first unit entails a non-trivial precondition of the 
second. We readily admit that presently we cannot offer a theoretically satisfying set of criteria to separate trivial and non-trivial preconditions, let alone a linguistic diagnostic for them. But we can characterize the difference informally by saying that preconditions that belong to many different types of events count as more trivial than conditions that belong to a small and specific set. For example, if we confine our attention to the actions of human beings, the condition that the agent of the event is alive conveys very little information about the event performed by the agent, since all actions done by humans require this. Therefore, even though (8b) entails the precondition that John was alive, we dismiss it as trivial and irrelevant. In contrast, the condition that, say, John has some money on him is required as a precondition by most everyday commercial transaction events which, however, constitute a relatively small subset of all the events that people may participate in as an agent. Notice that Hobbs's (1985) characterization of OCCASION also relies implicitly on such a distinction without which it could be trivially satisfied. ${ }^{3}$

Enthymematic entailment Having gone into a shop entails having been in the shop, but the latter does not entail per se that the initial state of buying a Coke holds. It is possible that the shop does not sell Coke or that the salesclerks have just decided to go on strike. These conditions would make it impossible for John to buy a Coke, even though he is in the shop. In view of such counterexamples, we might be inclined to conclude that the connection between the final state of the first DU and the initial state of the second DU cannot be entailment.

However, while it is true that logical entailment means that there is no possible world in which the premise is true and the conclusion is false, the word "entailment" (just like the word "inference" in Hobbs 1985) has a more flexible meaning. Proposition $\phi$ is said to enthymematically entail proposition $\psi$ if there is a nonempty set of propositions $\Phi$ such that $\Phi \cup\{\phi\}$ logically entails $\psi$. Thus, while being in the shop does not entail in a deductively valid way the conclusion that a non-trivial precondition of buying a Coke holds, it may entail it if an appropriate set of background propositions $\Phi$ is also taken into account.

These propositions may come from a wide variety of sources. For example, they may come from knowledge about how a normal shop is run: normally, the staff is

3 See also AI research on planning, which relies on such a distinction. AI planning theorists often appeal to initial states to model the coherence of dialogs (see, e.g., Litman \& Allen 1987). Moreover, they appeal to initial states to account for indirect speech act recognition, e.g., one interprets the question "Do you know the time?" as a request rather than yes-no question by way of an inference from an enabling pre-condition to the event itself (see, e.g., Perrault \& Allen 1980). Finally, initial states have been used to explain particular cases of pragmatic enrichment, e.g., why from "John went to the store and bought milk" one gets the enrichment that the milk was bought at the store, whereas the inference is not required as an answer to the question "What did John do today?". Thanks to an anonymous reviewer for directing us to this research. 
An argument for definitional adequacy of RESULT and NARRATION

at the counter and not on strike, normally, a shop sells a variety of common goods, and so on. Other propositions may come from the discourse itself: for example, we can infer from (8) that the shop mentioned in the discourse sells, among other things, Coke, and we can add to our stock of propositions this fact and whatever else it involves. ${ }^{4}$ Also, it is asserted that John bought and not stole the Coke which, again, allows us to add certain propositions to our set of (hidden) premises (e.g., that John gave some money to the clerk, John legally came into the possession of a drink, etc.).

Support Theories We propose that the entailment from the occurrence of the final state of the first DU to the occurrence of the initial state of the second DU is of the enthymematic type that we just described above, and we would like to connect it now with an issue that has been well known in theories of conditionals for a long time.

Bennett (2003) discusses what he calls "Support Theories" of the subjunctive conditional, originating with Goodman (1947), according to which a counterfactual conditional such as

(9) If you struck that match, it would light.

presupposes a huge set of tacitly assumed contingently true propositions such as "there is sufficient amount of oxygen around," "the air is still," "the match is dry enough," "you strike the match with sufficient force," "the matchbox is coated with enough phosphorus," etc. The conditional in (9) is true if this set of propositions, which Bennett (2003) calls Support, taken in conjunction with another set of propositions which Bennett calls Laws and which contains the causal laws and other relevant regularities reigning at the actual world, conjoined with the antecedent of the conditional validly entails the consequent of the conditional.

Notice that even though Goodman's (1947) proposal concerns counterfactual conditionals in the first place, the theory can be applied in the case of a simple indicative conditional as well, compare:

If you strike that match, it will light.

Arguably, the same background propositions can be assumed in this case as those assumed in the case of (9).

It is important that all the propositions in this tacitly assumed set Support are true. For example, the truth of the following conditional,

4 This is not much in the present case, but consider a shop in which John buys a considerable amount of pesticide for his farm-such shops are run very differently from a plain grocery store. 
If you make a noise, the guard will wake up.

requires, among other things, the truth of the proposition "the guard is not deaf". If the world in which (11) is evaluated does not satisfy this condition, then (11) will not be true in the evaluation world. In other words, the truth of the conditional depends both on the set of the tacit propositions introduced by the conditional and on what is actually the case in the world of evaluation.

As we will see shortly, the Support Theory of conditionals is very well suited to our goals in the present paper. ${ }^{5}$ To that end, we assume that a discourse $\sigma_{1} ; \sigma_{2}$ determines a set of (true) propositions $\mathscr{S}_{N}\left(\sigma_{1} ; \sigma_{2}\right)$, where the subscript $N$ is there to mark this set off from a different support set that we will introduce later on. The discourse relation between $\sigma_{1}$ and $\sigma_{2}$ is NARRATION just in case, taken together with the set of background propositions $\mathscr{S}_{N}\left(\sigma_{1} ; \sigma_{2}\right)$ and the set of relevant laws and regularities $L$, the proposition that the final state of the change of state inferable from the assertion of $\sigma_{1}$ occurs entails the proposition that the initial state of the change of state inferable from the assertion of $\sigma_{2}$ occurs.

This idea is spelled out below, in formula (1), where the predicate constant $\mathrm{O}(\cdot)$ applies to a term that denotes an eventuality, and the resulting atomic formula expresses the proposition that the eventuality in question occurs in the world of evaluation.

$$
\mathscr{S}_{N}\left(\sigma_{1} ; \sigma_{2}\right) \cup L \cup\left\{\mathrm{O}\left(\mathbf{s}\left(\sigma_{1}\right)\right)\right\} \models \mathrm{O}\left(\left\ulcorner_{\mathbf{s}}\left(\sigma_{2}\right)\right) .\right.
$$

Applying the deduction theorem to (1), we get:

$$
\mathscr{S}_{N}\left(\sigma_{1} ; \sigma_{2}\right) \cup L \models \mathrm{O}\left(\overline{\mathbf{s}}\left(\sigma_{1}\right)\right) \rightarrow \mathrm{O}\left(\left\ulcorner\mathbf{s}\left(\sigma_{2}\right)\right)\right.
$$

And now, we are in position to define NARRATION as follows:

Definition 4 (NARRATION-final version)

$$
\operatorname{NARRATION}\left(\sigma_{1}, \sigma_{2}\right) \stackrel{\text { def }}{\Longleftrightarrow} \mathscr{S}_{N}\left(\sigma_{1} ; \sigma_{2}\right) \cup L=\mathrm{O}\left(\mathbf{s}\left(\sigma_{1}\right)\right) \rightarrow \mathrm{O}\left(\left\ulcorner\mathbf{s}\left(\sigma_{2}\right)\right)\right.
$$

In the next section we extend this analysis to RESULT, which we propose involves two changes deduced from the DUs.

5 The Support Theory of conditionals is known to have problems which makes it an unlikely candidate for being the theory of conditionals in general. For example, the problem of identifying the propositions that are co-tenable with the antecedent of a counterfactual conditional is usually seen as involving a potentially vicious circularity (see Bennett 2003, but the problem was recognized already by Goodman (1947)). However, we will see in $\S 3.1$ that we apply the theory in a tightly controlled way only to derive certain constraints. 
An argument for definitional adequacy of RESULT and NARRATION

\subsection{The definition of RESULT}

Consider the following discourse (12) from Lascarides \& Asher (1993):

$$
\text { a. John pushed Max. }
$$

b. Max fell.

Here we have a clear intuition that the change described in (12a) somehow triggered the change described in (12b): the pushing by John resulted in the falling of Max.

Now consider the discourse (13), which we take to be on a par with (12):

a. John struck the match.

b. It lit.

Notice that this discourse is closely related to sentence (10), repeated below:

(10) If you strike that match, it will light.

We are going to capitalize on this connection below. Briefly, what we claim is that a discourse exemplifying RESULT can be interpreted as involving an enthymematic entailment, just like discourses with NARRATION, with one important caveat: the entailment with RESULT does not involve initial and final states but, rather, it involves the changes themselves that are inferred from the DUs.

In other words, what we propose is not very different from what we have already seen in Kehler's (2002) definition of RESULT in Definition 2, which we repeat below:

Definition 2 (RESULT by Kehler)

Given DUs $\sigma_{1}, \sigma_{2}, \operatorname{RESULT}\left(\sigma_{1}, \sigma_{2}\right)$ holds iff $P$ can be inferred from the assertion of $\sigma_{1}$ and $Q$ from $\sigma_{2}$, where normally $P \rightarrow Q$.

However, we also draw upon Hobbs's (1985) definition in that we preserve Hobbs's notion of change and use it to specify the propositional variables $P$ and $Q$ in Kehler's definition, and let the background propositions take care of the modifier "normally" in Definition 2. In particular, we assume (as before) that a discourse determines a support set of contingently true propositions which we denote by $\mathscr{S}_{R}\left(\sigma_{1} ; \sigma_{2}\right)$, where the subscript $R$ indicates that this set is relevant to the evaluation of RESULT. The discourse relation between $\sigma_{1}$ and $\sigma_{2}$ is RESULT just in case the proposition that the change $\mathbf{c}\left(\sigma_{1}\right)$ inferable from the assertion of $\sigma_{1}$ occurs, taken in conjunction with Support $\mathscr{S}_{R}\left(\sigma_{1} ; \sigma_{2}\right)$ and the relevant laws $L$, entails the proposition that the change $\mathbf{c}\left(\sigma_{2}\right)$ inferable from the assertion of $\sigma_{2}$ occurs. Formally:

$$
\mathscr{S}_{R}\left(\sigma_{1} ; \sigma_{2}\right) \cup L \cup\left\{\mathrm{O}\left(\mathbf{c}\left(\sigma_{1}\right)\right)\right\} \models \mathrm{O}\left(\mathbf{c}\left(\sigma_{2}\right)\right) .
$$


And, again by an application of the deduction theorem, we get:

$$
\mathscr{S}_{R}\left(\sigma_{1} ; \sigma_{2}\right) \cup L \models \mathrm{O}\left(\mathbf{c}\left(\sigma_{1}\right)\right) \rightarrow \mathrm{O}\left(\mathbf{c}\left(\sigma_{2}\right)\right) .
$$

Finally, on the basis of formula (4), we define RESULT as follows:

Definition 5 (RESULT-final version)

$$
\operatorname{RESULT}\left(\sigma_{1}, \sigma_{2}\right) \stackrel{\text { def }}{\Longleftrightarrow} \mathscr{S}_{R}\left(\sigma_{1} ; \sigma_{2}\right) \cup L \models \mathrm{O}\left(\mathbf{c}\left(\sigma_{1}\right)\right) \rightarrow \mathrm{O}\left(\mathbf{c}\left(\sigma_{2}\right)\right)
$$

\subsection{The relationship between RESULT and NARRATION}

Consider the discourses in (14a) and (15a) and a particular transformation thereof (henceforth: "ability explanation") involving the insertion of the modal expression 'be able to' and a 'because'-clause in (14b) and (15b):

a. Josef walked to the window. He opened it.

b. Josef was able to open the window because he walked up to it.

a. Josef picked up the book from the floor. He turned to pg. 20.

b. Josef was able to turn to pg. 20 because he picked up the book from the floor.

Based on the discussion in the previous section, we consider the discourses in (14a) and (15a) to exemplify NARRATION, and it appears that such discourses can always be used in ability explanations. This is expected from our definition of NARRATION since this relation involves bringing about the non-trivial preconditions that allow for the change entailed by the second DU, and ability attribution presupposes that such a possibility is available.

Importantly, discourses exemplifying RESULT can also be used in an ability explanation: ${ }^{6}$

a. John pushed the brake with all his force and stopped the car right at the edge of the precipice.

b. John was able to stop the car right at the edge of the precipice because he pushed the brake with all his force.

a. The red marble hit the white marble hard, and the white marble pushed the green marble off the table.

6 However, unlike NARRATION discourses, RESULT discourses cannot always be transformed into ability explanations, e.g., '??Max was able to fall because John pushed him.' Notice, however, that 'Max was able to fall' is quite odd on its own, so the infelicity is expected. This is probably a consequence of the fact that abilities are hard to attribute to non-agentive participants that simply undergo the event but do not control it. 
An argument for definitional adequacy of RESULT and NARRATION

b. The white marble was able to push the green marble off the table because the red marble had hit it hard.

Why should RESULT discourses entail an ability explanation that is characteristic of NARRATION? We propose to explain this pattern by saying that whenever RESULT holds between two DUs $\sigma_{1}$ and $\sigma_{2}$, then, provided that 'be able to' can felicitously be inserted in $\sigma_{2}$ (see footnote 6 ), $\sigma_{1}$ and $\sigma_{2}$ can be used in an ability explanation because NARRATION holds between $\sigma_{1}$ and $\sigma_{2}$ as well. Put briefly, RESULT entails NARRATION.

\section{Assumption 1 (RESULT and NARRATION)}

For any discourse $\sigma_{1} ; \sigma_{2}$,

if $\operatorname{RESULT}\left(\sigma_{1} ; \sigma_{2}\right)$, then NARRATION $\left(\sigma_{1} ; \sigma_{2}\right)$.

This assumption will play a pivotal role in the next section, where we derive constraints on possible EDUs via an abductive argument.

We end this section by noting that the entailment between RESULT and NARRATION is asymmetrical. Evidence for this comes from the observation that, unlike discourses exemplifying RESULT, discourses exemplifying NARRATION cannot usually be used in explanations without explicit reference to ability. For example, (14a) cannot be transformed into (18) (cf. (14b)):

(14a) Josef walked to the window. He opened it.

(18) \# Josef opened the window because he walked up to it.

Note that the oddness of (18) is not rooted in the incompatibility of either DU in an explanation. As the following examples show, both DUs can perfectly appear in the relevant position of a 'because'-clause:

a. Josef opened the window because Mary asked him to.

b. Josef startled the bird in the cage because he walked up to it.

The most reasonable explanation for the oddness of (18) is, therefore, that NARRATION between two DUs does not guarantee that RESULT also holds between them.

\section{Abducing structural constraints on EDUs}

The goal of the present section is to derive general constraints pertaining to the admissible structures underlying EDUs - defined in terms of initial/final states and the corresponding change-by a formal, abductive argument. We verify the plausibility of our derived EDUs by showing that they correspond to well attested English sentences. 


\subsection{Structural laws}

Given our proposed definitions of RESULT and NARRATION, Assumption 1, namely that RESULT entails NARRATION, is equivalent to the following claim:

$$
\text { If } \begin{aligned}
\mathscr{S}_{R}\left(\sigma_{1} ; \sigma_{2}\right) \cup L=\mathrm{O}\left(\mathbf{c}\left(\sigma_{1}\right)\right) \rightarrow & \mathrm{O}\left(\mathbf{c}\left(\sigma_{2}\right)\right) \text {, then } \\
& \mathscr{S}_{N}\left(\sigma_{1} ; \sigma_{2}\right) \cup L \models \mathrm{O}\left(\boldsymbol{\mathbf { s }}\left(\sigma_{1}\right)\right) \rightarrow \mathrm{O}\left(\left\ulcorner_{\mathbf{s}}\left(\sigma_{2}\right)\right) .\right.
\end{aligned}
$$

Notice that there is no guarantee whatsoever that $\mathscr{S}_{R}\left(\sigma_{1} ; \sigma_{2}\right)$ is the same set of propositions as $\mathscr{S}_{N}\left(\sigma_{1} ; \sigma_{2}\right)$ because the conditionals that determine these sets are different themselves, i.e., these support sets may change from discourse to discourse. Therefore, we can only guarantee the general validity of this entailment by relying on the set of laws L present in both definitions. In other words, we can ensure formula (5) and, by the same token, Assumption 1, if we assume that there is a set of special laws, or regularities, $\Delta \subseteq L$ such that

$$
\Delta \cup\left\{\mathrm{O}\left(\mathbf{c}\left(\sigma_{1}\right)\right) \rightarrow \mathrm{O}\left(\mathbf{c}\left(\sigma_{2}\right)\right)\right\} \models \mathrm{O}\left(\overline{\mathbf{s}}\left(\sigma_{1}\right)\right) \rightarrow \mathrm{O}\left(\left\ulcorner_{\mathbf{s}}\left(\sigma_{2}\right)\right)\right.
$$

holds. This set $\Delta$ of special laws pertains to the possible ways $\overline{\mathbf{s}}, \mathbf{s}$ and $\mathbf{c}$ may combine in the event structures associated with various DUs, given the limiting condition in formula (5). Therefore, in order to ensure Assumption 1, we need to find a set $\Delta$ of appropriate propositions that satisfies the entailment in formula (6).

Without imposing further constraints on $\Delta$, however, we would have infinitely many trivial or uninteresting solutions for $\Delta$, e.g., $\Delta=\left\{\mathrm{O}\left(\mathbf{c}\left(\sigma_{1}\right)\right) \wedge \neg \mathrm{O}\left(\mathbf{c}\left(\sigma_{1}\right)\right)\right\}$ or $\Delta=\left\{\mathrm{O}\left(\mathbf{s}\left(\sigma_{1}\right)\right) \rightarrow \mathrm{O}\left(\left\ulcorner_{\mathbf{s}}\left(\sigma_{2}\right)\right)\right\}\right.$, which would be of no use. To avoid this, we place the following constraints on the set of formulas $\Delta$ with respect to every discourse $\sigma_{1} ; \sigma_{2}$ :

C-1: Non-triviality: $\Delta \not \models \mathrm{O}\left(\mathbf{s}\left(\sigma_{1}\right)\right) \rightarrow \mathrm{O}\left(\varsigma^{\mathbf{s}}\left(\sigma_{2}\right)\right)$.

C-2: $\Delta$ must be minimal in the sense that

$$
\left\{\mathrm{O}\left(\mathbf{c}\left(\sigma_{1}\right)\right) \rightarrow \mathrm{O}\left(\mathbf{c}\left(\sigma_{2}\right)\right)\right\} \cup \Delta \models \mathrm{O}\left(\overline{\mathbf{s}}\left(\sigma_{1}\right)\right) \rightarrow \mathrm{O}\left(\left\ulcorner^{\mathbf{s}}\left(\sigma_{2}\right)\right)\right.
$$

but for all $\Delta^{\prime} \varsubsetneqq \Delta$,

$$
\left\{\mathrm{O}\left(\mathbf{c}\left(\sigma_{1}\right)\right) \rightarrow \mathrm{O}\left(\mathbf{c}\left(\sigma_{2}\right)\right)\right\} \cup \Delta^{\prime} \not \models \mathrm{O}\left(\mathbf{s}\left(\sigma_{1}\right)\right) \rightarrow \mathrm{O}\left(\Gamma_{\mathbf{s}}\left(\sigma_{2}\right)\right) .
$$

C-3: The elements of $\Delta$ must be homogeneous in the following sense: for any $\phi \in \Delta$, all occurrences of the variable $\sigma$ in $\phi$ must have the same index (i.e., either all of them must be ' 1 ', or all of them must be ' 2 ').

C-4: If $\phi \in \Delta$, then $\phi$ is not a literal (an atomic formula or its negation). 
Constraint $\mathrm{C}-1$ says that $\Delta$ in itself should not be sufficient to ensure the conclusion; without this constraint, NARRATION would automatically follow from the general background assumptions $\Delta$, which would trivialize the relation. The minimality constraint in C-2 is self-explanatory. Constraints C-3 and C-4, however, are not trivial and deserve a more detailed explanation.

The reason why we add Constraint C-3 to the set of constraints is closely related to the general goals of the present research, which is the formal derivation of principled connections between discourse relations and the underlying event structure. One way to turn this general idea into a specific research plan is to ask the following question: what kind of regularities do discourse relations require to obtain between the various components of an eventuality? By demanding that $\Delta$ be homogeneous in the sense explained in C-3 above, we limit our attention to the structural regularities that are internal to the eventualities inferable from the assertion of a DU.

The rationale behind Constraint $\mathrm{C}-4$ is also related to the general goals that we described above. The language that we use to formulate our definitions contains only one predicate constant, namely, $\mathrm{O}(\cdot)$, that can be applied to a term that denotes a state or a change, and the resulting atomic formula expresses the proposition that the eventuality in question occurs in the world of evaluation. Since $\Delta$ contains propositions that are assumed about the event structure in any discourse whatsoever, including such an atomic proposition (or its negation) in $\Delta$ would be equivalent to elevating the occurrence (or non-occurrence) of some state or change to the level of lawlike, necessary truths. But this would be absurd: for example, if $\Delta$ contained the proposition $\mathrm{O}(\mathbf{s}(\sigma))$, then the hearer would be justified to infer already from the general background knowledge that the final state related to the DUs that he or she is interpreting holds. This would trivialize the role played by $\Delta$, so we exclude this possibility in Constraint C-4.

Deriving $\Delta$ Let us transform the conjunction of the premise $\mathrm{O}\left(\mathbf{c}\left(\sigma_{1}\right)\right) \rightarrow \mathrm{O}\left(\mathbf{c}\left(\sigma_{2}\right)\right)$ and the negation of the conclusion $\neg\left(\mathrm{O}\left(\overrightarrow{\mathbf{s}}\left(\sigma_{1}\right)\right) \rightarrow \mathrm{O}\left({ }_{\mathbf{s}}\left(\sigma_{2}\right)\right)\right)$ into disjunctive normal form, yielding $\left(\neg \mathrm{O}\left(\mathbf{c}\left(\sigma_{1}\right)\right) \wedge \mathrm{O}\left(\overrightarrow{\mathbf{s}}\left(\sigma_{1}\right)\right) \wedge \neg \mathrm{O}\left(\Gamma_{\mathbf{s}}\left(\sigma_{2}\right)\right)\right) \vee\left(\mathrm{O}\left(\mathbf{c}\left(\sigma_{2}\right)\right) \wedge \mathrm{O}\left(\overrightarrow{\mathbf{s}}\left(\sigma_{1}\right)\right) \wedge\right.$ $\neg \mathrm{O}\left(\left\ulcorner\mathbf{s}\left(\sigma_{2}\right)\right)\right)$. We can make this formula contradictory by falsifying the disjuncts at the same time, which is the condition expressed by $\left(\mathrm{O}\left(\mathbf{c}\left(\sigma_{1}\right)\right) \vee \neg \mathrm{O}\left(\overrightarrow{\mathbf{s}}\left(\sigma_{1}\right)\right) \vee\right.$ $\mathrm{O}\left(\left\ulcorner\mathbf{s}\left(\sigma_{2}\right)\right)\right) \wedge\left(\neg \mathrm{O}\left(\mathbf{c}\left(\sigma_{2}\right)\right) \vee \neg \mathrm{O}\left(\overrightarrow{\mathbf{s}}\left(\sigma_{1}\right)\right) \vee \mathrm{O}\left(\left\ulcorner\mathbf{s}\left(\sigma_{2}\right)\right)\right)\right.$. But we are only interested in homogeneous, non-literal solutions, which leaves $\left(\mathrm{O}\left(\mathbf{c}\left(\sigma_{1}\right)\right) \vee \neg \mathrm{O}\left(\mathbf{s}\left(\sigma_{1}\right)\right)\right) \wedge$ $\left(\neg \mathrm{O}\left(\mathbf{c}\left(\sigma_{2}\right)\right) \vee \mathrm{O}\left(\left\ulcorner\mathbf{s}\left(\sigma_{2}\right)\right)\right)\right.$, i.e., $\left(\mathrm{O}\left(\mathbf{s}\left(\sigma_{1}\right)\right) \rightarrow \mathrm{O}\left(\mathbf{c}\left(\sigma_{1}\right)\right)\right) \wedge\left(\mathrm{O}\left(\mathbf{c}\left(\sigma_{2}\right)\right) \rightarrow \mathrm{O}\left(\left\ulcorner\mathbf{s}\left(\sigma_{2}\right)\right)\right)\right.$. This yields the set $\Delta$ of structural regularities pertaining to all possible $\sigma$ :

$$
\Delta=\left\{\mathrm{O}(\mathbf{c}(\sigma)) \rightarrow \mathrm{O}\left(\varsigma_{\mathbf{s}}(\sigma)\right), \mathrm{O}(\mathbf{s}(\sigma)) \rightarrow \mathrm{O}(\mathbf{c}(\sigma))\right\}
$$

The reader can check that this is indeed a solution for formula (6). 
Interpreting $\Delta$ Let us discuss the two formulas in $\Delta$ in turn. The first formula says that if the occurrence of a change of state $\mathbf{c}(\sigma)$ can be inferred from the assertion of a DU, then the occurrence of a state $5 \mathbf{s}(\sigma)$ can also be inferred from it; that is, the occurrence of this state is a precondition of the occurrence of the change.

At this point, the reader might suspect a vicious circularity lurking beneath the surface, and it is important to allay this suspicion, reminding the reader of the special nature of this investigation. Note that even though we have informally characterized an initial state as the situation whose occurrence is a (non-trivial) precondition of the change inferable from the unit, we have never incorporated this into the formal system, which only consists of the two definitions and the assumption concerning the logical relationship between them. The fact that the symbol ' $\mathrm{s}(\sigma)$ ' must denote an initial state has been derived from our definitions of RESULT and NARRATION and the constraint that the former must entail the latter through purely formal manipulations of these postulates. In other words, this information about the general constitution of event structures is implicit in the concepts of RESULT and NARRATION suggested by Hobbs (1985) and Kehler (2002), and what we have done is make it explicit through conceptual analysis.

Turning to the second formula in $\Delta$, we can paraphrase it as saying that the occurrence of the state $\mathbf{s}(\sigma)$ inferable from $\sigma$ implies the occurrence of a change $\mathbf{c}(\sigma)$ inferable from $\sigma$. Note that this claim is weaker than what our informal characterization of $\mathbf{S}(\sigma)$ involves. Recall that when we informally introduced $\mathbf{s}(\sigma)$, we intended it to be the (linguistically relevant) final state of a change of states inferable from a unit $\sigma$. The set of postulates consisting of our definitions and the relationship between them is not strong enough to justify this-we have only established a weaker result, namely, that $\mathbf{S}(\sigma)$ cannot occur without a change $\mathbf{c}(\sigma)$ occurring. Even though this is compatible with the interpretation according to which $\mathbf{S}(\sigma)$ is the final state of the change in question, it does not, strictly speaking, entail that interpretation without additional postulates. We will not attempt to derive such postulates in the present paper but, rather, turn to the question how the propositions in $\Delta$ constrain the possible event structures that serve as the interpretations of DUs.

\subsection{Possibilities for EDUs}

The previous subsection discussed the results of our abductive argument, namely the following derived set of propositions:

$$
\Delta=\left\{\mathrm{O}(\mathbf{c}(\sigma)) \rightarrow \mathrm{O}\left(\Gamma_{\mathbf{s}}(\sigma)\right), \mathrm{O}(\mathbf{s}(\sigma)) \rightarrow \mathrm{O}(\mathbf{c}(\sigma))\right\}
$$

The propositions in $\Delta$ contain a DU variable $\sigma$ that is bound by an implicit universal quantifier. Given our definitions of RESULT and NARRATION, for the entailment 
An argument for definitional adequacy of RESULT and NARRATION

\begin{tabular}{|l|c|c|}
\hline Structure & Admissible? & Example \\
\hline \hline$\langle-,-,-\rangle$ & yes & - \\
$\langle\mathbf{s},-,-\rangle$ & yes & $(20)$ \\
$\langle\mathbf{s}, \mathbf{c},-\rangle$ & yes & $(21 \mathrm{a}),(21 \mathrm{~b})$ \\
$\langle\mathbf{s}, \mathbf{c}, \mathbf{s}\rangle$ & yes & $(22 \mathrm{a}),(22 \mathrm{~b})$ \\
$\langle-, \mathbf{c},-\rangle$ & no & - \\
$\langle-, \mathbf{c}, \mathbf{s}\rangle$ & no & - \\
$\langle-,-, \mathbf{s}\rangle$ & no & - \\
$\langle\mathbf{s},-, \mathbf{s}\rangle$ & no & - \\
\hline
\end{tabular}

Table 2 Admissible DU structures

from $\operatorname{RESULT}\left(\sigma_{1}, \sigma_{2}\right)$ to NARRATION $\left(\sigma_{1}, \sigma_{2}\right)$ to be valid, all DUs $\sigma$ must observe the constraints in $\Delta$.

Let us assume that a DU is interpreted in terms of "what can be inferred from it" ( $c f$. Definitions 1 and 2). In particular, we make the following assumption concerning a minimal ontology: ${ }^{7}$

Assumption 2 (Minimal ontology for DUs)

The interpretation of an arbitrary DU $\sigma$ is a triple of the form $\left\langle{ }^{\mathbf{s}}(\sigma), \mathbf{c}(\sigma), \mathbf{s}(\sigma)\right\rangle$, where $\overline{\mathbf{s}}(\sigma)$ and $\mathbf{S}(\sigma)$ are states, and $\mathbf{c}(\sigma)$ is a change.

Crucially, we do not require that all three components be present in the interpretation of every DU. On the contrary, even though at first we will assume that every logical combination is available to fill in the places for the components, in this section we will derive various constraints that narrow down the eight a priori possibilities to three.

Table 2 shows the possibilities that the constraints in $\Delta$ permit or forbid with respect to EDUs. The possibilities in Table 2 are those that our analysis of the two discourse relations-RESULT and NARRATION-forces us to postulate, given the constraints in $\Delta$. This explains why a particular abstract structure may be matched with several radically different English examples in Table 2: what we have derived from RESULT and NARRATION is too coarse-grained to differentiate between the

7 If the present ontology seems somewhat cumbersome, then we ask the reader to consider the following fact: in many current event ontologies one finds, among other things, states as well as events (see, e.g., Higginbotham 1985, Parsons 1990, Kamp \& Reyle 1993, Landman 2000, Rothstein 2004, Bittner 2007). If we look at the ontological category of changes as a very impoverished version of the ontological category of events, then the ontology that we assume in this paper is not very different from the ontology that many researchers assume-in fact, it is a very simple version thereof. 
subtypes of DUs falling under the same abstract structure.

In order to show that these possibilities are not absurd, we have matched each admissible EDU to an actual sentence of English, shown below, in (20)-(22), which we discuss in turn. ${ }^{8}$

(20) The door is locked.

(21) a. Tim walked in the park yesterday morning.

b. Bill winked.

(22) a. Last year, Mary built a house.

b. John arrived a few minutes ago.

The sentence in (20) expresses a state of the door. We associate the structure $\langle\overline{\mathbf{s}},-,-\rangle$ with stative EDUs. In order to see why, let us consider how to interpret this representation. In particular: does the identity of the slots occupied or left empty in these representations convey any substantial information? We would like to suggest that the answer is 'no', and that the structures assigned to stative EDUs in Table 2 are fundamentally different from the other representations in the table.

We have assumed in $\S 3.2$ that the interpretation of a DU is an ordered sequence of ontological kinds. However, ordering makes sense only if there are at least two things to order. So while, e.g., $\left\langle\Gamma_{\mathbf{s}}, \mathbf{c},-\right\rangle$ makes perfect sense, what does $\left\langle{ }^{\mathbf{s}},-,-\right\rangle$ mean? How is it different from $\langle-,-, \mathbf{s}\rangle$ ? Recall that initial states and final states are not two different kinds of states but simply states: the only difference between them is where they occur in the structure. But if there is no other element in the structure, then we have hardly more than a structure consisting of a single state simpliciter.

As for (21a), here we see an activity predicate in the simple past tense that has a 'bounded' interpretation. Note that we can infer the occurrence of a series of changes in Tim's spatial position from (21a), and this kind of inference is always appropriate with activities. Indeed, since at least Dowty (1979), activities have been analyzed as describing a process that is decomposed into a series of smaller changes. We could model this idea by thinking of our change component $\mathbf{c}(\sigma)$ as being a set (or series) of changes. As we shall now see, this is something we need to assume on independent grounds, namely to model the difference between accomplishments and achievements.

With respect to (21b), we can infer from it that there was a change, and also that it had some non-trivial preconditions (such as that Bill's eye was open), but we cannot infer any linguistically relevant postconditions. Note that the analysis of (21a) and (21b) are parallel, except that (21b) has a single associated change

8 Although it does not have linguistic relevance, we have included the structure $\langle-,-,-\rangle$ in Table 2 because our constraints do not exclude it from the possibilities. 
An argument for definitional adequacy of RESULT and NARRATION

whereas (21a) has a series of such changes ( $c f$. Rothstein 2008).

The sentences in (22) contain accomplishments and achievements, respectively. While there is plenty of empirical evidence that there are linguistically relevant distinctions between these predicates, and presumably an event structure should reflect these differences, recall that our goal is to simply show that our derived DUs are plausible. To that end, it is plausible that the truth of a DU like (22a) depends on some non-trivial precondition (e.g., the architect drew out the plan), and a change component, which (as noted above with respect to activities) corresponds to series of changes, as well as a final state, namely the house being built; this state is entailed by (22a). The same analysis is plausible for (22b) with one caveat: (22b) differs from (22a) in describing a single change.

\section{Conclusion}

A\&L proposed to account for narrative progression by appealing to two different flavors of causality, encoded by RESULT and NARRATION. In this paper we argued that NARRATION can be extracted from Hobbs's (1985) OCCASION, while RESULT can be built on a refined version of Kehler's (2002) characterization of this relation. In particular, we redefined NARRATION and RESULT in terms of enthymematic entailment, inspired by the Support Theories of conditionals. Based on our definitions, along with the idea that RESULT and NARRATION stand in a strict logical relationship, we offered a novel way for testing the adequacy of our definitions. In particular, we derived general constraints pertaining to possible EDUs-defined in terms of final/initial states and the corresponding change-by a formal, abductive argument. We verified the plausibility of our derived EDUs by showing that they correspond to well attested English sentences. An outstanding question, currently pursued in Altshuler \& Varasdi (In review), is to see what we predict when we concatenate our derived EDUs, and in particular, whether we make the correct predictions with respect to RESULT and NARRATION. Moreover, in the future, we hope to apply the methodology advocated here to define and test other discourse relations with temporal import, such as BACKGROUND, EXPLANATION, ELABORATION and PARALLEL.

\section{References}

Altshuler, Daniel \& Károly Varasdi. (In review) Discourse relations, possible discourse units and narrative progression.

Asher, Nicholas \& Alex Lascarides. 2003. Logics of Conversation. Cambridge, England: Cambridge University Press.

Bennett, Jonathan. 2003. A Philosophical Guide to Conditionals. Oxford University Press. doi:10.1093/0199258872.001.0001. 
Bittner, Maria. 2007. Online Update: Temporal, modal, and de se anaphora in polysynthetic discourse. In Chris Barker \& Pauline Jacobson (eds.), Direct Compositionality, 363-404. Oxford: Oxford University Press.

Dowty, David. 1979. Word Meaning and Montague Grammar. Dordrecht: Reidel. doi:10.1007/978-94-009-9473-7.

Goodman, Nelson. 1947. The problem of counterfactual conditionals. Journal of Philosophy 44(5). 113-128. doi:10.2307/2019988.

Higginbotham, Jim. 1985. On semantics. Linguistic Inquiry (16). 547-593.

Hobbs, Jerry. 1985. On the coherence and structure of discourse. Technical Report CSLI-85-37. Center for the Study of Language and Information, Stanford University, Stanford, California.

Hobbs, Jerry R. 1990. Literature and Cognition. Stanford, California: Center for the Study of Language and Information.

Hume, David. 2008. An Enquiry Concerning Human Understanding: And Selections from a Treatise of Human Nature. New York, NY: Cosimo Classics.

Kamp, Hans \& Uwe Reyle. 1993. From Discourse to Logic: Introduction to Modeltheoretic Semantics of Natural Language, Formal Logic and Discourse Representation Theory. Kluwer Academic Publishers. doi:10.1007/978-94-0171616-1. Studies in Linguistics and Philosophy, Volume 42.

Kehler, Andrew. 2002. Coherence, reference and the theory of grammar. Stanford, California: Center for the Study of Language and Information.

Landman, Fred. 2000. Events and Plurality. Dordrecht: Kluwer. doi:10.1007/97894-011-4359-2.

Lascarides, Alex \& Nicholas Asher. 1993. Temporal interpretation, discourse relations, and common sense entailment. Linguistics and Philosophy 16(5). 437-495. doi:10.1007/BF00986208.

Litman, Diane J. \& James F. Allen. 1987. A plan recognition model for subdialogues in conversation. Cognitive Science (11). 163-200. doi:10.3115/980491.980554.

Moens, Marc \& Mark Steedman. 1988. Temporal ontology and temporal reference. Computational Linguistics 14(2). 15-29.

Parsons, Terence. 1990. Events in the Semantics of English: A Study in Subatomic Semantics. Cambridge, Massachusetts: MIT Press.

Perrault, Raymond \& James F. Allen. 1980. A plan-based analysis of indirect speech acts. American Journal of Computational Linguistics (6). 167-182.

Rothstein, Susan. 2004. Structuring Events: A Study in the Semantics of Lexical Aspect. Wiley-Blackwell. doi:10.1002/9780470759127.

Rothstein, Susan. 2008. Two puzzles for a theory of lexical aspect: semelfactives and degree achievements. In Johannes Dölling, Tatjana Heyde-Zybatow \& Martin Schäfer (eds.), Event Structures in Linguistic Form and Interpretation, 175-198. Walter de Gruyter. doi:10.1515/9783110925449.175. 